\title{
Strategic planning and performance measurement using Fuzzy DEMATEL: A case study of Iran Kaolin and Barite company
}

\author{
Abolfazl Danaei ${ }^{a}$, Mohammad Foroughi Jabbari ${ }^{\mathrm{b}}$ and Leila Omidifard ${ }^{\mathrm{a}^{*}}$ \\ ${ }^{a}$ Department of Management, Semnan branch, Islamic Azad University, Semnan, Iran \\ ${ }^{b}$ Faculty of Engineering, Semnan branch, Islamic Azad University, Semnan, Iran

\section{CHRON I C LE} \\ Article history: \\ Received January 18, 2013 \\ Received in revised format \\ 10 May 2013 \\ Accepted May 152013 \\ Available online \\ May 162013 \\ Keywords: \\ Ranking \\ FUzzy DEMATEL

\section{A B S T R A C T} \\ This paper presents an empirical investigation to measure the performance of a mining firm in \\ province of Semnan, Iran based on fuzzy fuzzy Decision Making Trial and Evaluation \\ Laboratory (DEMATEL) technique. The proposed study of this paper uses strength, weakness, \\ opportunity and threat (SWOT) technique to analyze the firm and using DEMATEL rank \\ various items based on their relative importance. Based on the results of our survey, cost \\ reduction and increase investment in mining sector are the most important components of this \\ survey. The study also compares the results with similar study, which has recently been \\ accomplished and we believe the proposed model is capable of detecting possible threats and \\ helping us provide possible actions.
}

SWOT
(C) 2013 Growing Science Ltd. All rights reserved.

\section{Introduction}

Many managerial decisions are involved with a set of different criteria where we often see some sorts of conflict among them leading us to use multi-criteria decision making (MCDM) techniques. Existing methods of equity investment, such as fundamental analysis, technical analysis, and institutional investor analysis, explore essential factors of stock price behaviors. Lee et al. (2011) provided the first analysis on the interactive relationships among the factors in incorporating the techniques of Decision Making Trial and Evaluation Laboratory (DEMATEL) (Fontela \& Gabus, 1974) and Analytic Network Process (ANP) (Saaty, 1994, 2004). They reported that factors from the existing analytical methodologies had significant interactive and self-feedback dynamics. Among the key factors, profitability has been the most important one influencing investment decision, followed by growth and trading volume. In addition, due to the complexity of the ANP, this study proposed a new method to simplify the process, and empirical evidences indicated that the approach was effective and efficient.

*Corresponding author. Tel: +989380358679

E-mail addresses: omidifardleila@yahoo.com (L.Omidifard)

C) 2013 Growing Science Ltd. All rights reserved.

doi: $10.5267 /$ j.ds1.2013.05.003 
According to Lin and $\mathrm{Wu}$ (2008) Causal analysis largely impacts the effectiveness of decisionmaking and the productivity of actions. The complicated relationship between cause and effect as well as the fuzzy nature of human judgment often make the casual analysis more difficult. Lin and $\mathrm{Wu}$ (2008) developed a fuzzy DEMATEL method for group decision-making to gather group ideas and analyzed the cause-effect relationship of complex problems in fuzzy environments. They used the fuzzy DEMATEL procedures and separated the involved criteria of a system into the cause and effect groups to help decision-makers concentrate on those criteria, which provide important impact. They presented an empirical study based on their proposed fuzzy DEMATEL method to the R\&D project selection of a Taiwanese firm. They reported that, within the cause group, the criterion of "probability of technical success" was the most important items for R\&D project selection, whereas the "strategic fit" and "potential size of market" have the best impact on the other criteria.

Tzeng et al. (2007) proposed a new hybrid MCDM model to address the independent relationship of evaluation criteria with the help of factor analysis and the dependent relationship of evaluation criteria with the aid of DEMATEL. They used analytical hierarchy process (AHP) (Saaty \& Vargas, 1994) and the fuzzy integral techniques (Li, 1999) for synthetic utility in accordance with subjective perception environment. Empirical results indicated that the proposed model was capable of producing effective evaluation of e-learning programs with adequate criteria that fit with respondent's perception patterns, especially when the evaluation criteria were numerous and intertwined. Zhou et al. (2011) discussed on how to enhance emergency management by segmenting complex impacting factors into groups to improve them in a stepwise way. To address this concern involving the vagueness of human judgments, they used an efficient technique that mixes fuzzy logic and DEMATEL. Considering the interdependence among factors, this fuzzy DEMATEL technique formed a structural model and then visualizes the causal relationships among factors through a causeeffect relationship diagram. Then based on the results of their proposed method, critical success factor (CSF) of emergency management was detected. They identified five factors out of 20 ones where all factors could be achieved in a stepwise way for better promoting the effectiveness and efficiency of emergency management.

Yang et al. (2008) proposed a novel hybrid MCDM model combined with DEMATEL and ANP to handle the dependence and feedback problems to suit the real-world applications. They also gave an instance to show the proposed method with applications thereof. Their results demonstrated the proposed method is more suitable for real-world applications than the traditional ones. Opricovic and Tzeng (2003) proposed fuzzy MCDM (FMCDM), which consists of two phases. In the first phase, the CFCS phase, the method converts the Fuzzy data into Crisp Scores, and the MCDM phase, MCDM. The model seems to be applicable for defuzzification within the MCDM model with a mixed set of crisp and fuzzy criteria. The CFCS method is based on the procedure of determining the left and right scores by fuzzy min and fuzzy max, respectively, and the total score is computed a weighted average based on the membership functions.

\section{The proposed model}

The proposed model of this paper uses fuzzy DEMATEL for ranking various strategies extracted SWOT matrix (Lee \& Walsh, 2011). In this section, we present details of the implementation of our proposed method.

Step 1. Setup goals and objectives,

Step 2. Determine fuzzy goals and scales based on the following triangular linguistic verbal terms, 


\begin{tabular}{ccccc}
\hline Very high $(\mathrm{VH})$ & High & Low & Very Low & No effect (NO) \\
\hline$(0.75,1,1)$ & $(0.5,0.75,1)$ & $(0.25,0.5,0.75)$ & $(0.0,0.25,0.50)$ & $(0.0,0.0,0.25)$ \\
\hline
\end{tabular}

Step 3. Setup decision matrix as follows,

$$
\tilde{Z}^{\langle k\rangle}=\left[\begin{array}{cccc}
0 & \tilde{Z}_{12}^{\langle k\rangle} & \cdots & \tilde{Z}_{1 n}^{\langle k\rangle} \\
\tilde{Z}_{21}^{\langle k\rangle} & 0 & \cdots & \tilde{Z}_{2 n}^{\langle k\rangle} \\
\vdots & \vdots & \cdots & \vdots \\
\tilde{Z}_{n 1}^{\langle k\rangle} & \tilde{Z}_{n 2}^{\langle k\rangle} & \cdots & 0
\end{array}\right] \quad k=1,2, \ldots, p
$$

where $\tilde{Z}_{i j}^{\langle k\rangle}=\left(\lambda_{i j}^{(k)}, m_{i j}^{\langle k\rangle}, u_{i j}^{(k)}\right)$ is associated with $k^{t h}$ decision maker, which is called Initial direct-relation fuzzy matrix.

Step 4. Normalize decision making matrix as follows,

$$
\begin{aligned}
\tilde{a}_{i}^{\langle k\rangle} & =\sum_{j=1}^{n} \tilde{Z}_{i j}^{\langle k\rangle}=\left(\sum_{j=1}^{n} \lambda_{i j}^{\langle k\rangle}, \sum_{j=1}^{n} m_{i j}^{\langle k\rangle}, \sum_{j=1}^{n} u_{i j}^{\langle k\rangle}\right), \\
r^{\langle k\rangle} & =\max _{1 \leq i \leq n}\left(\sum_{j=1}^{n} u_{i j}^{\langle k\rangle}\right) .
\end{aligned}
$$

Now, in order to convert scale criteria into measurable scales we use linearization technique. Let $\tilde{X}^{\langle k\rangle}$ be relationship matrix associated with $k^{\text {th }}$ decision maker, which is defined as follows,

$$
\tilde{X}^{\langle k\rangle}=\left[\begin{array}{cccc}
\tilde{X}_{11}^{\langle k\rangle} & \tilde{X}_{12}^{\langle k\rangle} & \cdots & \tilde{X}_{1 n}^{\langle k\rangle} \\
\tilde{X}_{21}^{\langle k\rangle} & \tilde{X}_{22}^{\langle k\rangle} & \cdots & \tilde{X}_{2 n}^{\langle k\rangle} \\
\vdots & \vdots & \cdots & \vdots \\
\tilde{X}_{n 1}^{\langle k\rangle} & \tilde{X}_{n 2}^{\langle k\rangle} & \cdots & \tilde{X}_{n n}^{\langle k\rangle}
\end{array}\right] \quad k=1,2, \ldots, p
$$

where

$$
\tilde{X}_{i j}^{\langle k\rangle}=\frac{\tilde{Z}_{i j}^{\langle k\rangle}}{r^{\langle k\rangle}}=\left(\frac{\lambda_{i j}^{\langle k\rangle}}{r^{\langle k\rangle}}, \frac{m_{i j}^{\langle k\rangle}}{r^{\langle k\rangle}}, \frac{u_{i j}^{\langle k\rangle}}{r^{\langle k\rangle}}\right)
$$

Like standard DEMATEL we assume there is at least one $i$ where $\sum_{j=1}^{n} u_{i j}^{\langle k\rangle}\left\langle r^{\langle k\rangle}\right.$.

Step 5. In order to compute total relation fuzzy matrix we first need to make sure that $\underset{w \rightarrow \infty}{\operatorname{Lim}} \tilde{X}^{w}=0$. Consider $\tilde{X}_{i j}=\left(\lambda_{i j}, m_{i j}, u_{i j}\right)$ and consider the following three matrices,

$$
X_{u}=\left[\begin{array}{cccc}
0 & u_{12} & \cdots & u_{1 n} \\
u_{21} & 0 & \cdots & u_{2 n} \\
\vdots & \vdots & \cdots & \vdots \\
u_{n 1} & u_{n 2} & \cdots & 0
\end{array}\right] \quad X_{m}=\left[\begin{array}{cccc}
0 & m_{12} & \cdots & m_{1 n} \\
u_{21} & 0 & \cdots & m_{2 n} \\
\vdots & \vdots & \cdots & \vdots \\
m_{n 1} & m_{n 2} & \cdots & 0
\end{array}\right] \quad X_{\lambda}=\left[\begin{array}{cccc}
0 & \lambda_{12} & \cdots & \lambda_{1 n} \\
\lambda_{21} & 0 & \cdots & \lambda_{2 n} \\
\vdots & \vdots & \cdots & \vdots \\
\lambda_{n 1} & \lambda_{n 2} & \cdots & 0
\end{array}\right]
$$

Like deterministic DEMATEL we define the following, 
$\tilde{T}=\operatorname{Lim}_{w \rightarrow \infty}\left(\tilde{X}+\tilde{X}^{2}+\ldots+\tilde{X}^{w}\right)=X \times(I-X)^{-1}$

Lemma 1. Let $\tilde{T}=\left[\begin{array}{cccc}\tilde{t}_{11} & \tilde{t}_{12} & \cdots & \tilde{t}_{1 n} \\ \tilde{t_{21}} & \tilde{t}_{22} & \cdots & \tilde{t}_{2 n} \\ \vdots & \vdots & \cdots & \vdots \\ \tilde{t}_{n 1} & \tilde{t}_{n 2} & \cdots & \tilde{t}_{n n}\end{array}\right]$ where $\tilde{t_{i j}}=\left(\lambda_{i j}^{\prime \prime}, m_{i j}^{\prime \prime}, u_{i j}^{\prime \prime}\right)$ then

$\operatorname{Matrix}\left[\lambda_{i j}^{\prime \prime}\right]=X_{\lambda} \times\left(I-X_{\lambda}\right)^{-1} \quad \operatorname{Matrix}\left[m_{i j}^{\prime \prime}\right]=X_{m} \times\left(I-X_{m}\right)^{-1} \quad \operatorname{Matrix}\left[u_{i j}^{\prime \prime}\right]=X_{u} \times\left(I-X_{u}\right)^{-1}$

Let $\quad \tilde{n}_{k}=\left(\lambda_{k}, m_{k}, u_{k}\right) ; k=1,2, \ldots, n, \quad$ where $\quad \tilde{n}_{k}^{\text {def }}$ is a crisp value, $L=\min \left(\lambda_{k}\right)$ and $R=\max \left(u_{k}\right) ; k=1,2, \ldots, n$ and $\Delta=R-L$ then we have,

$$
\tilde{n}_{k}^{\text {def }}=L+\Delta \times \frac{(m-L)(\Delta+u-m)^{2}(R-\lambda)+(u-L)^{2}(\Delta+m-\lambda)^{2}}{(\Delta+m-\lambda)(\Delta+u-m)^{2}(R-\lambda)+(u-L)(\Delta+u-m)}
$$

\section{Case study}

The proposed study of this paper is implemented for one of Iranian firm called Iran Kaolin and Barite company located in province of Semnan, Iran. Iran Kaolin and Barite company was established in 1992 and it produces and provides mineral products needed by industries such as oil and gas drilling, chemical and ceramic. The company is one of major producers of industrial mineral powders such as Barite, Bentonite and Hematite according to API, OCMA \& ASTM standards, micronized calcium carbonate, Kaolin, Talc, Feldespar, Silicaflour and other mineral powders. The firm is also active in exporting its products to the countries in the Persian Gulf region and CIS countries. Table 1 demonstrates the summary of SWOT for our study.

Table 1

The summary of SWOT

\begin{tabular}{llc}
\hline & \multicolumn{1}{c}{ Strength $(\mathrm{S})$} & \multicolumn{1}{c}{ Weakness $(\mathrm{W})$} \\
\hline \multirow{3}{*}{ Opportunities $(\mathrm{O})$} & \multicolumn{1}{c}{ Using S-O strategies } & Using W-O strategies \\
\cline { 2 - 3 } & Using competitive strategies $\left(\mathrm{O}_{2}, \mathrm{O}_{3}, \mathrm{~S}_{2}\right)$ & Increase investment in production $\left(\mathrm{W}_{1}, \mathrm{O}_{3}\right)$ \\
& \multicolumn{1}{c}{ Increase customer satisfaction $\left(\mathrm{O}_{5}, \mathrm{~S}_{3}\right)$} & Increase investment in mining $\left(\mathrm{W}_{2}, \mathrm{O}_{2}\right)$ \\
& & Use competitors' capability to increase production $\left(\mathrm{W}_{1}, \mathrm{O}_{4}\right)$ \\
\cline { 2 - 3 } Threats $(\mathrm{T})$ & Use present good reputation $\left(\mathrm{S}_{2}, \mathrm{~T}_{1}\right)$ & Using W-T strategies \\
& Increase global opportunities $\left(\mathrm{T}_{1}, \mathrm{~S}_{3}, \mathrm{~S}_{4}, \mathrm{~S}_{6}\right)$ & Concentration on export methods $\left(\mathrm{W}_{1}, \mathrm{~T}_{4}\right)$ \\
& Increase export $\left(\mathrm{T}_{4}, \mathrm{~S}_{2}\right)$ & \\
& Reduce production cost $\left(\mathrm{T}_{5}, \mathrm{~S}_{4}, \mathrm{~S}_{6}\right)$ & \\
\hline
\end{tabular}

In order to accomplish the SWOT strategies, we need to ask decision makers to express their opinions in terms of verbal language. Table 2 to Table 5 demonstrate details of our findings as follows,

Table 2

S-O strategies

\begin{tabular}{|c|c|c|c|c|c|}
\hline & Description & $\mathrm{O} 2$ & $\mathrm{O} 3$ & $\mathrm{O} 4$ & O5 \\
\hline S2 & Existence of sales' records for domestic and international firms & $\mathrm{H}$ & $\mathrm{VH}$ & $\mathrm{NO}$ & $\mathrm{NO}$ \\
\hline S3 & Existence of necessary test equipment and access to ISO 9001:2000 & NO & NO & $\mathrm{NO}$ & $\mathrm{VH}$ \\
\hline S4 & Existence of mines, which are located only $1.5 \mathrm{~km}$ away from firm & $\mathrm{H}$ & $\mathrm{H}$ & $\mathrm{H}$ & NO \\
\hline S6 & Existence of export opportunities towards north part of Iran & NO & $\mathrm{H}$ & $\mathrm{NO}$ & NO \\
\hline $\mathrm{O} 2$ & Unique product characteristics and lack of replacement product & 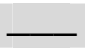 & - & - & 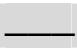 \\
\hline $\mathrm{O} 3$ & Existence of common resources in the region & - & - & - & 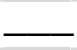 \\
\hline $\mathrm{O} 4$ & Quitting one of the biggest competitors from the region & $\ldots$ & - & 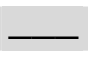 & - \\
\hline $\mathrm{O5}$ & Existence of international standards to control the quality & $\bar{\square}$ & $\overline{-}$ & - & - \\
\hline
\end{tabular}


Table 3

S-T strategies

\begin{tabular}{llccc}
\hline \multicolumn{2}{l}{ Description } & T1 & T4 & T5 \\
\hline S2 & Existence of sales' records to well-known firms & H & NO & H \\
S3 & Existence of necessary test equipment and access to ISO 9001:2000 & H & NO & VH \\
S4 & Existence of mines, which are located only 1.5 km away from firm & L & NO & L \\
S6 & Existence of export opportunities towards north part of Iran & M & H & NO \\
T1 & Tension in south region & - & - & - \\
T4 & Economic crises and shortage in liquidity & - & - \\
T5 & Unfair completion through newly established firms in other countries & - & - \\
\hline
\end{tabular}

Table 4

$\mathrm{W}-\mathrm{O}$ strategies

\begin{tabular}{clcc}
\hline & Description & W1 & W2 \\
\hline O2 & Lack of replacement products & NO & L \\
O3 & Existence of common natural resources in the region and competition & L & NO \\
O4 & Quitting one of the biggest competitors from the region & $\mathrm{H}$ & NO \\
O5 & Existence of necessary test equipment and access to ISO 9001:2000 & NO & NO \\
W1 & Production limit in quantity & - & - \\
W2 & Insufficient investment in mineral resources & - & - \\
\hline
\end{tabular}

\section{Table 5}

W-T strategies

\begin{tabular}{clcc}
\hline \multicolumn{2}{l}{ Description } & W1 & W2 \\
\hline T1 & Tension in south region & NO & NO \\
T4 & Economic crises and shortage in liquidity & M & NO \\
T5 & Unfair completion through newly established firms in other countries & NO & NO \\
W1 & Production limit in quantity & - & - \\
W2 & Insufficient investment in mineral resources & - & - \\
\hline
\end{tabular}

Table 6 summarizes the results of relationships between different actions.

Table 6

The summary of relationship between various components

\begin{tabular}{ccccccccccccccc}
\hline Component & S2 & S3 & S4 & S6 & O2 & O3 & O4 & O5 & T1 & T4 & T5 & W1 & W2 \\
\hline S2 & - & - & - & - & H & VH & NO & NO & H & NO & H & NO & NO \\
S3 & - & - & - & - & NO & NO & NO & VH & H & NO & VH & NO & NO \\
S4 & - & - & - & - & H & H & H & NO & L & NO & L & NO & NO \\
S6 & - & - & - & - & NO & H & NO & NO & M & H & NO & NO & NO \\
O2 & NO & NO & NO & NO & - & - & - & - & NO & NO & NO & NO & L \\
O3 & NO & NO & NO & NO & - & - & - & - & NO & NO & NO & L & NO \\
O4 & NO & NO & NO & NO & - & - & - & - & NO & NO & NO & H & NO \\
O5 & NO & NO & NO & NO & - & - & - & - & NO & NO & NO & NO & NO \\
T1 & NO & NO & NO & NO & NO & NO & NO & NO & - & - & - & NO & NO \\
T4 & NO & NO & NO & NO & NO & NO & NO & NO & - & - & - & M & NO \\
T5 & NO & NO & NO & NO & NO & NO & NO & NO & - & - & - & NO & NO \\
W1 & NO & NO & NO & NO & NO & NO & NO & NO & NO & NO & NO & - & - \\
W2 & NO & NO & NO & NO & NO & NO & NO & NO & NO & NO & NO & - & - \\
\hline
\end{tabular}

Now we are able to compute $X_{\lambda}$ for different constructive components of various strategies. 
Table 7

The summary for $X_{\lambda}$

\begin{tabular}{|c|c|c|c|c|c|c|c|c|c|c|c|c|c|}
\hline Component & $\mathrm{S} 2$ & S3 & $\mathrm{S} 4$ & S6 & $\mathrm{O} 2$ & $\mathrm{O} 3$ & O4 & O5 & $\mathrm{T} 1$ & $\mathrm{~T} 4$ & T5 & W1 & W2 \\
\hline S2 & 0 & 0 & 0 & 0 & 0.5 & 0.75 & 0 & 0 & 0.5 & 0 & 0.5 & 0 & 0 \\
\hline $\mathrm{S} 3$ & 0 & 0 & 0 & 0 & 0 & 0 & 0 & 0.75 & 0.5 & 0 & 0.75 & 0 & 0 \\
\hline S4 & 0 & 0 & 0 & 0 & 0.5 & 0.5 & 0.5 & 0 & 0.25 & 0 & 0.25 & 0 & 0 \\
\hline S6 & 0 & 0 & 0 & 0 & 0 & 0.5 & 0 & 0 & 0.25 & 0.5 & 0 & 0 & 0 \\
\hline $\mathrm{O} 2$ & 0 & 0 & 0 & 0 & 0 & 0 & 0 & 0 & 0 & 0 & 0 & 0 & 0.25 \\
\hline $\mathrm{O} 3$ & 0 & 0 & 0 & 0 & 0 & 0 & 0 & 0 & 0 & 0 & 0 & 0.25 & 0 \\
\hline $\mathrm{O} 4$ & 0 & 0 & 0 & 0 & 0 & 0 & 0 & 0 & 0 & 0 & 0 & 0.5 & 0 \\
\hline O5 & 0 & 0 & 0 & 0 & 0 & 0 & 0 & 0 & 0 & 0 & 0 & 0 & 0 \\
\hline $\mathrm{T} 1$ & 0 & 0 & 0 & 0 & 0 & 0 & 0 & 0 & 0 & 0 & 0 & 0 & 0 \\
\hline $\mathrm{T} 4$ & 0 & 0 & 0 & 0 & 0 & 0 & 0 & 0 & 0 & 0 & 0 & 0.25 & 0 \\
\hline T5 & 0 & 0 & 0 & 0 & 0 & 0 & 0 & 0 & 0 & 0 & 0 & 0 & 0 \\
\hline W1 & 0 & 0 & 0 & 0 & 0 & 0 & 0 & 0 & 0 & 0 & 0 & 0 & 0 \\
\hline W2 & 0 & 0 & 0 & 0 & 0 & 0 & 0 & 0 & 0 & 0 & 0 & 0 & 0 \\
\hline
\end{tabular}

Table 8

The summary for $X_{m}$

\begin{tabular}{|c|c|c|c|c|c|c|c|c|c|c|c|c|c|}
\hline Component & $\mathrm{S} 2$ & S3 & S4 & S6 & $\mathrm{O} 2$ & $\mathrm{O} 3$ & O4 & O5 & T1 & $\mathrm{T} 4$ & T5 & W1 & W2 \\
\hline $\mathrm{S} 2$ & 0 & 0 & 0.75 & 1 & 0 & 0 & 0.75 & 0 & 0.75 & 0 & 0 & 0 & 0 \\
\hline S3 & 0 & 0 & 0 & 0 & 0 & 1 & 0.75 & 0 & 1 & 0 & 0 & 0 & 0 \\
\hline S4 & 0 & 0 & 0.75 & 0.75 & 0.75 & 0 & 0.5 & 0 & 0.5 & 0 & 0 & 0 & 0 \\
\hline S6 & 0 & 0 & 0 & 0.75 & 0 & 0 & 0.5 & 0.75 & 0 & 0 & 0 & 0 & 0 \\
\hline $\mathrm{O} 2$ & 0 & 0 & 0 & 0 & 0 & 0 & 0 & 0 & 0 & 0 & 0.5 & 0 & 0 \\
\hline $\mathrm{O} 3$ & 0 & 0 & 0 & 0 & 0 & 0 & 0 & 0 & 0 & 0.5 & 0 & 0 & 0 \\
\hline O4 & 0 & 0 & 0 & 0 & 0 & 0 & 0 & 0 & 0 & 0.75 & 0 & 0 & 0 \\
\hline O5 & 0 & 0 & 0 & 0 & 0 & 0 & 0 & 0 & 0 & 0 & 0 & 0 & 0 \\
\hline $\mathrm{T} 1$ & 0 & 0 & 0 & 0 & 0 & 0 & 0 & 0 & 0 & 0 & 0 & 0 & 0 \\
\hline $\mathrm{T} 4$ & 0 & 0 & 0 & 0 & 0 & 0 & 0 & 0 & 0 & 0.5 & 0 & 0 & 0 \\
\hline T5 & 0 & 0 & 0 & 0 & 0 & 0 & 0 & 0 & 0 & 0 & 0 & 0 & 0 \\
\hline W1 & 0 & 0 & 0 & 0 & 0 & 0 & 0 & 0 & 0 & 0 & 0 & 0 & 0 \\
\hline W2 & 0 & 0 & 0 & 0 & 0 & 0 & 0 & 0 & 0 & 0 & 0 & 0 & 0 \\
\hline
\end{tabular}

Table 9

The summary for $X_{u}$

\begin{tabular}{|c|c|c|c|c|c|c|c|c|c|c|c|c|c|}
\hline Component & $\mathrm{S} 2$ & $\mathrm{~S} 3$ & $\mathrm{~S} 4$ & S6 & $\mathrm{O} 2$ & $\mathrm{O} 3$ & $\mathrm{O} 4$ & $\mathrm{O} 5$ & $\mathrm{~T} 1$ & $\mathrm{~T} 4$ & $\mathrm{~T} 5$ & $\mathrm{~W} 1$ & W2 \\
\hline S2 & 0 & 0.25 & 0.25 & 0.25 & 1 & 1 & 0.25 & 0.25 & 1 & 0.25 & 1 & 0.25 & 0.25 \\
\hline S3 & 0.25 & 0 & 0.25 & 0.25 & 0.25 & 0.25 & 0.25 & 1 & 1 & 0.25 & 1 & 0.25 & 0.25 \\
\hline S4 & 0.25 & 0.25 & 0 & 0.25 & 1 & 1 & 1 & 0.25 & 0.75 & 0.25 & 0.75 & 0.25 & 0.25 \\
\hline S6 & 0.25 & 0.25 & 0.25 & 0 & 0.25 & 1 & 0.25 & 0.25 & 0.75 & 1 & 0.25 & 0.25 & 0.25 \\
\hline $\mathrm{O} 2$ & 0.25 & 0.25 & 0.25 & 0.25 & 0 & 0.25 & 0.25 & 0.25 & 0.25 & 0.25 & 0.25 & 0.25 & 0.75 \\
\hline $\mathrm{O} 3$ & 0.25 & 0.25 & 0.25 & 0.25 & 0.25 & 0 & 0.25 & 0.25 & 0.25 & 0.25 & 0.25 & 0.75 & 0.25 \\
\hline O4 & 0.25 & 0.25 & 0.25 & 0.25 & 0.25 & 0.25 & 0 & 0.25 & 0.25 & 0.25 & 0.25 & 1 & 0.25 \\
\hline O5 & 0.25 & 0.25 & 0.25 & 0.25 & 0.25 & 0.25 & 0.25 & 0 & 0.25 & 0.25 & 0.25 & 0.25 & 0.25 \\
\hline $\mathrm{T} 1$ & 0.25 & 0.25 & 0.25 & 0.25 & 0.25 & 0.25 & 0.25 & 0.25 & 0 & 0.25 & 0.25 & 0.25 & 0.25 \\
\hline $\mathrm{T} 4$ & 0.25 & 0.25 & 0.25 & 0.25 & 0.25 & 0.25 & 0.25 & 0.25 & 0.25 & 0 & 0.25 & 0.75 & 0.25 \\
\hline T5 & 0.25 & 0.25 & 0.25 & 0.25 & 0.25 & 0.25 & 0.25 & 0.25 & 0.25 & 0.25 & 0 & 0.25 & 0.25 \\
\hline W1 & 0.25 & 0.25 & 0.25 & 0.25 & 0.25 & 0.25 & 0.25 & 0.25 & 0.25 & 0.25 & 0.25 & 0 & 0.25 \\
\hline W2 & 0.25 & 0.25 & 0.25 & 0.25 & 0.25 & 0.25 & 0.25 & 0.25 & 0.25 & 0.25 & 0.25 & 0.25 & 0 \\
\hline
\end{tabular}

Table 10

The summary of Matrix $\left[\lambda_{i j}^{\prime \prime}\right]=X_{\lambda} \times\left(I-X_{\lambda}\right)^{-1}$

\begin{tabular}{|c|c|c|c|c|c|c|c|c|c|c|c|c|c|}
\hline Component & S2 & S3 & $\mathrm{S} 4$ & S6 & $\mathrm{O} 2$ & $\mathrm{O} 3$ & $\mathrm{O} 4$ & O5 & $\mathrm{T} 1$ & $\mathrm{~T} 4$ & T5 & W1 & W2 \\
\hline $\mathrm{S} 2$ & 0 & 0 & 0 & 0 & 0.5 & 0.75 & 0 & 0 & 0.5 & 0 & 0.5 & 0.188 & 0.125 \\
\hline S3 & 0 & 0 & 0 & 0 & 0 & 0 & 0 & 0.75 & 0.5 & 0 & 0.75 & 0 & 0 \\
\hline S4 & 0 & 0 & 0 & 0 & 0.5 & 0.5 & 0.5 & 0 & 0.25 & 0 & 0.25 & 0.375 & 0.125 \\
\hline S6 & 0 & 0 & 0 & 0 & 0 & 0.5 & 0 & 0 & 0.25 & 0.5 & 0 & 0.25 & 0 \\
\hline $\mathrm{O} 2$ & 0 & 0 & 0 & 0 & 0 & 0 & 0 & 0 & 0 & 0 & 0 & 0 & 0.25 \\
\hline $\mathrm{O} 3$ & 0 & 0 & 0 & 0 & 0 & 0 & 0 & 0 & 0 & 0 & 0 & 0.25 & 0 \\
\hline O4 & 0 & 0 & 0 & 0 & 0 & 0 & 0 & 0 & 0 & 0 & 0 & 0.5 & 0 \\
\hline O5 & 0 & 0 & 0 & 0 & 0 & 0 & 0 & 0 & 0 & 0 & 0 & 0 & 0 \\
\hline $\mathrm{T} 1$ & 0 & 0 & 0 & 0 & 0 & 0 & 0 & 0 & 0 & 0 & 0 & 0 & 0 \\
\hline $\mathrm{T} 4$ & 0 & 0 & 0 & 0 & 0 & 0 & 0 & 0 & 0 & 0 & 0 & 0.25 & 0 \\
\hline T5 & 0 & 0 & 0 & 0 & 0 & 0 & 0 & 0 & 0 & 0 & 0 & 0 & 0 \\
\hline W1 & 0 & 0 & 0 & 0 & 0 & 0 & 0 & 0 & 0 & 0 & 0 & 0 & 0 \\
\hline W2 & 0 & 0 & 0 & 0 & 0 & 0 & 0 & 0 & 0 & 0 & 0 & 0 & 0 \\
\hline
\end{tabular}


Table 11

The summary of Matrix $\left[m_{i j}^{\prime \prime}\right]=X_{m} \times\left(I-X_{m}\right)^{-1}$

\begin{tabular}{|c|c|c|c|c|c|c|c|c|c|c|c|c|c|}
\hline Component & $\mathrm{S} 2$ & $\mathrm{~S} 3$ & $\mathrm{~S} 4$ & S6 & $\mathrm{O} 2$ & $\mathrm{O} 3$ & $\mathrm{O} 4$ & O5 & $\mathrm{T} 1$ & $\mathrm{~T} 4$ & T5 & W1 & $\mathrm{W} 2$ \\
\hline $\mathrm{S} 2$ & 0 & 0 & 0 & 0 & 0.75 & 1 & 0 & 0 & 0.75 & 0 & 0.75 & 0.5 & 0.375 \\
\hline S3 & 0 & 0 & 0 & 0 & 0 & 0 & 0 & 1 & 0.75 & 0 & 1 & 0 & 0 \\
\hline S4 & 0 & 0 & 0 & 0 & 0.75 & 0.75 & 0.75 & 0 & 0.5 & 0 & 0.5 & 0.938 & 0.375 \\
\hline S6 & 0 & 0 & 0 & 0 & 0 & 0.75 & 0 & 0 & 0.5 & 0.75 & 0 & 0.75 & 0 \\
\hline $\mathrm{O} 2$ & 0 & 0 & 0 & 0 & 0 & 0 & 0 & 0 & 0 & 0 & 0 & 0 & 0.5 \\
\hline $\mathrm{O} 3$ & 0 & 0 & 0 & 0 & 0 & 0 & 0 & 0 & 0 & 0 & 0 & 0.5 & 0 \\
\hline $\mathrm{O} 4$ & 0 & 0 & 0 & 0 & 0 & 0 & 0 & 0 & 0 & 0 & 0 & 0.75 & 0 \\
\hline O5 & 0 & 0 & 0 & 0 & 0 & 0 & 0 & 0 & 0 & 0 & 0 & 0 & 0 \\
\hline $\mathrm{T} 1$ & 0 & 0 & 0 & 0 & 0 & 0 & 0 & 0 & 0 & 0 & 0 & 0 & 0 \\
\hline T4 & 0 & 0 & 0 & 0 & 0 & 0 & 0 & 0 & 0 & 0 & 0 & 0.5 & 0 \\
\hline T5 & 0 & 0 & 0 & 0 & 0 & 0 & 0 & 0 & 0 & 0 & 0 & 0 & 0 \\
\hline W1 & 0 & 0 & 0 & 0 & 0 & 0 & 0 & 0 & 0 & 0 & 0 & 0 & 0 \\
\hline W2 & 0 & 0 & 0 & 0 & 0 & 0 & 0 & 0 & 0 & 0 & 0 & 0 & 0 \\
\hline
\end{tabular}

Table 12

The summary of Matrix $\left[u_{i j}^{\prime \prime}\right]=X_{u} \times\left(I-X_{u}\right)^{-1}$

\begin{tabular}{cccccccccccccccc}
\hline Component & S2 & S3 & S4 & S6 & O2 & O3 & O4 & O5 & T1 & T4 & T5 & W1 & W2 \\
\hline S2 & -0.355 & -0.155 & -0.155 & -0.155 & -0.139 & -0.045 & -0.248 & -0.248 & -0.014 & -0.248 & -0.076 & -0.385 & -0.1 \\
S3 & -0.112 & -0.312 & -0.112 & -0.112 & -0.246 & -0.314 & -0.179 & -0.301 & -0.144 & -0.179 & -0.189 & -0.417 & -0.211 \\
S4 & -0.178 & -0.178 & -0.378 & -0.178 & -0.089 & -0.017 & -0.196 & -0.284 & -0.213 & -0.284 & -0.142 & -0.181 & -0.142 \\
S6 & -0.123 & -0.123 & -0.123 & -0.323 & -0.271 & -0.135 & -0.197 & -0.197 & -0.05 & -0.283 & -0.32 & -0.074 & -0.232 & -0.13 \\
O2 & -0.056 & -0.056 & -0.056 & -0.056 & -0.323 & -0.157 & -0.09 & -0.09 & -0.168 & -0.09 & -0.146 & -0.208 & -0.215 \\
O3 & -0.056 & -0.056 & -0.056 & -0.056 & -0.123 & -0.357 & -0.09 & -0.09 & -0.168 & -0.09 & -0.146 & -0.112 & -0.105 \\
O4 & -0.064 & -0.064 & -0.064 & -0.064 & -0.141 & -0.179 & -0.302 & -0.102 & -0.192 & -0.102 & -0.166 & -0.242 & -0.12 \\
O5 & -0.04 & -0.04 & -0.04 & -0.04 & -0.088 & -0.112 & -0.064 & -0.264 & -0.12 & -0.064 & -0.104 & -0.149 & -0.075 \\
T1 & -0.04 & -0.04 & -0.04 & -0.04 & -0.088 & -0.112 & -0.064 & -0.064 & -0.32 & -0.064 & -0.104 & -0.149 & -0.075 \\
T4 & -0.056 & -0.056 & -0.056 & -0.056 & -0.123 & -0.157 & -0.09 & -0.09 & -0.168 & -0.29 & -0.146 & -0.112 & -0.105 \\
T5 & -0.04 & -0.04 & -0.04 & -0.04 & -0.088 & -0.112 & -0.064 & -0.064 & -0.12 & -0.064 & -0.304 & -0.149 & -0.075 \\
W1 & -0.04 & -0.04 & -0.04 & -0.04 & -0.088 & -0.112 & -0.064 & -0.064 & -0.12 & -0.064 & -0.104 & -0.349 & -0.075 \\
W2 & -0.04 & -0.04 & -0.04 & -0.04 & -0.088 & -0.112 & -0.064 & -0.064 & -0.12 & -0.064 & -0.104 & -0.149 & -0.275 \\
\hline
\end{tabular}

Next, we build triangular numbers based on instruction gave in Step 5 of the proposed method.

Table 13

The summary of triangular numbers

\begin{tabular}{|c|c|c|c|c|c|c|c|c|c|c|c|c|c|c|}
\hline Component & S2 & S3 & S4 & S6 & $\mathrm{O} 2$ & $\mathrm{O} 3$ & $\mathrm{O} 4$ & $\mathrm{O} 5$ & $\mathrm{~T} 1$ & T4 & T5 & W1 & $\mathrm{W} 2$ & $\mathrm{R}$ \\
\hline S2 & -0.032 & -0.014 & -0.014 & -0.014 & -0.184 & -0.635 & -0.022 & -0.022 & -0.483 & -0.022 & 3.915 & -0.026 & -0.008 & 3.157 \\
\hline S3 & -0.01 & -0.028 & -0.01 & -0.01 & -0.022 & -0.028 & -0.016 & -0.275 & -0.161 & -0.016 & 15.316 & -0.038 & -0.019 & 14.682 \\
\hline S4 & -0.016 & -0.016 & -0.034 & -0.016 & 1.029 & -0.353 & -0.041 & -0.026 & -0.016 & -0.026 & -0.027 & -0.17 & -0.014 & -0.652 \\
\hline S6 & -0.011 & -0.011 & -0.011 & -0.029 & -0.025 & -0.201 & -0.018 & 0.018 & -0.059 & -0.028 & -0.029 & -0.126 & -0.021 & -0.161 \\
\hline $\mathrm{O} 2$ & -0.005 & -0.005 & -0.005 & -0.005 & -0.029 & -0.014 & -0.008 & -0.008 & -0.015 & -0.008 & -0.013 & -0.019 & -0.036 & -0.1 \\
\hline $\mathrm{O} 3$ & -0.005 & -0.005 & -0.005 & -0.005 & -0.0 & -0.0 & -0.008 & & -0.015 & -0.008 & -0.013 & & -0.01 & -0.112 \\
\hline $\mathrm{O} 4$ & -0.006 & -0.006 & -0.006 & -0.006 & -0.013 & -0.016 & -0.027 & -0.009 & -0.017 & -0.009 & -0.015 & -0.004 & -0.011 & -0.137 \\
\hline O5 & -0.004 & -0.004 & -0.004 & -0.004 & -0.008 & -0.01 & -0.006 & -0.024 & -0.011 & -0.006 & -0.009 & -0.013 & -0.007 & -0.109 \\
\hline $\mathrm{T} 1$ & -0.004 & -0.004 & -0.004 & -0.004 & -0.008 & -0.01 & -0.006 & & -0.029 & -0.006 & -0.009 & & -0.007 & -0.109 \\
\hline $\mathrm{T} 4$ & -0.005 & -0.005 & -0.005 & -0.005 & -0.011 & -0.014 & -0.008 & -0.008 & -0.015 & -0.026 & -0.013 & -0.014 & -0 . & -0.112 \\
\hline T5 & -0.004 & -0.004 & -0.004 & -0.004 & -0.008 & -0.01 & -0.006 & -0.006 & -0.011 & -0.006 & -0.028 & -0.013 & -0.007 & -0.109 \\
\hline W1 & -0.004 & -0.004 & -0.004 & -0.004 & -0.008 & -0.01 & -0.006 & -0.006 & -0.011 & -0.006 & -0.009 & -0.0 & -0.007 & -0.109 \\
\hline W2 & & -0.004 & -0.004 & -0.004 & -0.008 & -0.01 & -0.006 & & & -0.006 & -0.009 & -0.013 & -0.025 & -0.109 \\
\hline $\mathrm{J}$ & -0.109 & -0.109 & -0.109 & -0.109 & 1.363 & -0.632 & -0.179 & -0.422 & -0.262 & -0.117 & 11.279 & -0.16 & -0.109 & - \\
\hline
\end{tabular}

Table 14

The summary of DEMATEL computations

\begin{tabular}{|c|c|c|c|c|}
\hline Strategies & (R) & $(\mathrm{J})$ & (R-J) & $(\mathrm{R}+\mathrm{J})$ \\
\hline S2 & 0.241 & -0.109 & 0.349 & 0.132 \\
\hline S3 & 0.191 & -0.109 & 0.3 & 0.083 \\
\hline S4 & 0.163 & -0.109 & 0.272 & 0.055 \\
\hline S6 & 0.166 & -0.109 & 0.275 & 0.058 \\
\hline $\mathrm{O} 2$ & 0.19 & -1.363 & 1.553 & -1.173 \\
\hline O3 & 0.213 & 0.632 & -0.418 & 0.845 \\
\hline O4 & 0.158 & -0.179 & 0.337 & -0.021 \\
\hline O5 & 0.22 & -0.422 & 0.642 & -0.202 \\
\hline $\mathrm{T} 1$ & 0.231 & 0.262 & $0.031-$ & 0.492 \\
\hline $\mathrm{T} 4$ & 0.225 & -0.117 & 0.342 & 0.108 \\
\hline T5 & 0.214 & 11.279 & -11.065 & 11.493 \\
\hline W1 & 0.31 & 0.16 & 0.15 & 0.47 \\
\hline W2 & 0.177 & -0.109 & 0.287 & 0.068 \\
\hline
\end{tabular}


Finally, we may present details of suitable strategies for the proposed case study of this paper in Table 15 as follows,

Table 15

The summary of appropriate strategies

\begin{tabular}{lcccc}
\hline Strategies & $(\mathrm{R})$ & $(\mathrm{J})$ & $(\mathrm{R}-\mathrm{J})$ & $(\mathrm{R}+\mathrm{J})$ \\
\hline Use strong competitive skills & 0.644 & -0.84 & 1.484 & -0.196 \\
\hline Increase customers' trust in products and services & 0.411 & -0.531 & 0.942 & -0.12 \\
\hline Take advantage of good reputations among existing customers & 0.472 & 0.153 & 0.319 & 0.625 \\
\hline Increase in competition capabilities in international market & 0.751 & -0.065 & 0.816 & 0.686 \\
Increase transaction with foreign customers & 0.466 & -0.226 & 0.692 & 0.24 \\
\hline Cost reduction endeavors & $\mathbf{0 . 5 4 3}$ & $\mathbf{1 1 . 0 6 1}$ & $\mathbf{- 1 0 . 5 1 8}$ & $\mathbf{1 1 . 6 0 4}$ \\
\hline Increase investment in production units & 0.523 & 0.792 & -0.269 & 1.315 \\
\hline Increase investment in mining industries & $\mathbf{0 . 3 6 7}$ & $\mathbf{- 1 . 4 7 2}$ & $\mathbf{1 . 8 3 9}$ & $\mathbf{- 1 . 1 0 5}$ \\
\hline Take advantage of competitors' capabilities to increase production & 0.468 & -0.019 & 0.487 & 0.449 \\
Allocate production for export purposes & 0.535 & 0.043 & 0.492 & 0.578 \\
\hline
\end{tabular}

As we can observe from the results of Table 15, cost reduction and increase investment in mining sector are the most important components of this survey.

\section{Discussion and conclusion}

In this paper, we have presented an empirical investigation to determine weakness, strength, opportunities and possible threats (SWOT) for a case study of an Iranian firm named Kaolin and Barite company, which was doing business on providing mineral products needed by industries such as oil and gas drilling, chemical and ceramic. They discussed different issues influencing the market activities using SWOT analysis and then ranked important components based on fuzzy DEMATEL. Based on the results of our survey, cost reduction and increase investment in mining sector are the most important components of this survey. The implementation of DEMATEL for ranking different alternatives has recently become popular among researchers (Shepherd \& Günter, 2006; Amiri et al., 2011).

Najmi and Makui (2010), for instance, provided hierarchical approach for measuring supply chain performance using AHP and DEMATEL methodologies. Sofiyabadi et al. (2012) presented an integrated balanced score card combined with DEMATEL technique to prioritize different alternatives for supply chain implementation.

Danaei and Omidifard (2013) performed strategic planning and performance measurement using balanced scorecard (BSC) (Kaplan \& Norton, 1992, 1996, 2004) for the same case study. The results of their survey indicated that the firm was able to make a $30 \%$ improvement on its performance after one-year implementation of BSC. In other words, the firm was able to make 59\% improvement on learning and growth, $33 \%$ growth on internal process, $32 \%$ growth on customer and $21 \%$ improvement on financial figures.

We hope the results of this survey along with other investigations accomplished recently could help this industry grow faster and build better business activities.

\section{Acknowledgment}

The authors would like to thank the anonymous referees for constructive comments on earlier version of this paper. 


\section{References}

Amiri, M., Sadaghiyani, J., Payani, N \& Shafieezadeh, M. (2011). Developing a DEMATEL method to prioritize distribution centers in supply chain. Management Science Letters, 1(3), 279-288.

Danaei, A., \& Omidifard, A. (2013). Strategic planning and performance measurement using balanced scorecard: A case study of Iran Kaolin and Barite company. Management Science Letters, 3(6). DOI: 10.5267/j.msl.2013.05.022.

Fontela, E. \& Gabus, A. (1974). DEMATEL. Progress Achieved, Futures, 6, 329-333.

Hunger, J. D., \& Wheelen, T. L. (2003). Essentials of strategic management. New Jersey: Prentice Hall.

Kaplan, R.S. \& Norton, D.P. (1992). The balanced scorecard - measures that drive performance, Harvard Business Review, 70(1), 71-79.

Kaplan, R.S. and Norton, D.P. (1996). The balanced scorecard: translating strategy into action. Harvard Business School Press, Boston, MA.

Kaplan, R.S. \& Norton, D.P. (2004), Strategy maps: converting intangible assets into tangible outcomes, Harvard Business School Press, Boston, MA.

Lee, S. F., \& Ko, A. S. O. (2000). Building balanced scorecard with SWOT analysis, and implementing "Sun Tzu's The Art of Business Management Strategies" on QFD methodology. Managerial Auditing Journal, 15(1/2), 68-76.

Lee, S., \& Walsh, P. (2011). SWOT and AHP hybrid model for sport marketing outsourcing using a case of intercollegiate sport. Sport Management Review, 14(4), 361-369.

Lee, W. S., Huang, A. Y., Chang, Y. Y., \& Cheng, C. M. (2011). Analysis of decision making factors for equity investment by DEMATEL and Analytic Network Process. Expert Systems with Applications, 38(7), 8375-8383.

Li, R. J. (1999). Fuzzy method in group decision making. Computers \& Mathematics with Applications, 38(1), 91-101.

Lin, C. J., \& Wu, W. W. (2008). A causal analytical method for group decision-making under fuzzy environment. Expert Systems with Applications, 34(1), 205-213.

Najmi, A \& Makui, A. (2010). Providing hierarchical approach for measuring supply chain performance using AHP and DEMATEL methodologies. International Journal of Industrial Engineering Computations, 1(2), 199-212.

Opricovic, S., \& Tzeng, G. H. (2003). Defuzzification within a multicriteria decision model. International Journal of Uncertainty, Fuzziness and Knowledge-Based Systems, 11(05), 635-652.

Yang, Y. P. O., Shieh, H. M., Leu, J. D., \& Tzeng, G. H. (2008). A novel hybrid MCDM model combined with DEMATEL and ANP with applications. International Journal of Operations Research, 5(3), 160-168.

Saaty, T. L. \& Vargas, L. (1994). Decision making with analytic hierarchy process. RWS.

Saaty, T. L. (1996). The analytic network process-decision making with dependence and feedback. Pittsburgh, PA: RWS Publications.

Saaty, T. L. (2004). The analytic network process: Dependence and feedback in decision making (Part 1): Theory and validation examples, SESSION 4B: Theory and development of the analytic hierarchy process/analytic network process, In The 17th International Conference on Multiple Criteria Decision Making, August 6-11, 2004 at The Whistler Conference Centre, Whistler, British Columbia, Canada.

Sofiyabadi, J., movahedi, M \& Nasab, S. (2012). Strategic orientation in evaluation of supply chain activities. Management Science Letters , 2(5), 1785-1794.

Shepherd, C., \& Günter, H. (2006). Measurement supply chain performance: current research and future directions. International Journal of Productivity and Performance management, 55(314), 242-258.

Tzeng, G. H., Chiang, C. H., \& Li, C. W. (2007). Evaluating intertwined effects in e-learning 
programs: A novel hybrid MCDM model based on factor analysis and DEMATEL. Expert Systems with Applications, 32(4), 1028-1044.

Zhou, Q., Huang, W., \& Zhang, Y. (2011). Identifying critical success factors in emergency management using a fuzzy DEMATEL method. Safety Science, 49(2), 243-252. 